\title{
Independent UP-algebras
}

\author{
Aiyared lampan ${ }^{\mathrm{a}, *}$, Pongpun Julatha $^{\mathrm{b}}$, Pannawit Khamrot ${ }^{\mathrm{c}}$, Daniel A. Romano ${ }^{\mathrm{d}}$ \\ ${ }^{a}$ Fuzzy Algebras and Decision-Making Problems Research Unit, Department of Mathematics, School of Science, University of Phayao, \\ Mae Ka, Mueang, Phayao 56000, Thailand. \\ ${ }^{b}$ Department of Mathematics, Faculty of Science and Technology, Pibulsongkram Rajabhat University, Phitsanulok 65000, Thailand. \\ ${ }^{c}$ Faculty of Science and Agricultural Technology, Rajamangala University of Technology Lanna Phitsanulok, Phitsanulok 65000, \\ Thailand. \\ ${ }^{d}$ International Mathematical Virtual Institute, Kordunaška Street, 78000 Banja Luka, Bosnia and Herzegovina.
}

\begin{abstract}
In this paper, we introduce the concept of a new algebraic structure: independent UP-algebras (in short, IUP-algebras), which is independent of UP-algebras. We also introduce the concepts of IUP-subalgebras, IUP-filters, IUP-ideals, and strong IUP-ideals of IUP-algebras and investigate their properties and relationships. Finally, we discuss the concept of homomorphisms between IUP-algebras and also study the direct and inverse images of four special subsets.
\end{abstract}

Keywords: UP-algebra, IUP-algebra, IUP-subalgebra, IUP-filter, IUP-ideal, strong IUP-ideal.

2020 MSC: 06F35, 03G25, 94B05.

(C)2022 All rights reserved.

\section{Introduction and preliminaries}

Among many algebraic structures, algebras of logic form an important class of algebras. Examples of these are BE-algebras [16], pseudo-BE-algebras [4], eBE-algebras [22], pseudo-eBE-algebras [29], pseudoCI-algebras [23], eGE-algebras [3], PSRU-algebras [34], KU-algebras [21], UP-algebras [9], UP-bialgebras [18], extension of KU/UP-algebras [24], and others, which are inspired by BCK-algebras [13] and BCIalgebras [14]. They are strongly connected with logic. The study and research on various algebraic structures are extensive, varied, and ongoing by many algebraists around the world, as we can see from the current number of articles citations based on Google Scholar (as of January 2022), for example, BCKalgebras have been cited more than 1000 times, BCI-algebras have been cited more than 700 times, BEalgebras have been cited more than 180 times, KU-algebras have been cited more than 160 times, and UP-algebras have been cited more than 100 times.

\footnotetext{
*Corresponding author

Email addresses: aiyared.ia@up.ac.th (Aiyared Iampan), pongpun.j@psru.ac.th (Pongpun Julatha), pk_g@rmutl.ac.th (Pannawit Khamrot), bato49@hotmail.com (Daniel A. Romano)
}

doi: $10.22436 /$ jmcs.027.01.06

Received: 2021-11-03 Revised: 2021-11-16 Accepted: 2022-01-19 
The goal of this study is to introduce the concept of a new algebraic structure: IUP-algebras, which is independent of UP-algebras. The concepts of IUP-subalgebras, IUP-filters, IUP-ideals, and strong IUPideals of IUP-algebras are also introduced. We look into some of their characteristics and qualities. Finally, we look at IUP-algebra homomorphisms and direct and inverse images of four special subsets.

Before we begin our study, let's review the definitions of KU-algebras, UP-algebras, and PU-algebras.

Definition 1.1 ([21]). An algebra $A=(A, 0,0)$ of type $(2,0)$ is called a KU-algebra, where $A$ is a nonempty set, $\circ$ is a binary operation on $A$, and 0 is a fixed element of $A$ (i.e., a nullary operation) if it satisfies the following axioms:

$$
\begin{aligned}
& (\text { for all } x, y, z \in A)((x \circ y) \circ((y \circ z) \circ(x \circ z))=0), \\
& \text { (for all } x \in A)(0 \circ x=x), \\
& \text { (for all } x \in A)(x \circ 0=0) \text {, and } \\
& (\text { for all } x, y \in A)((x \circ y=0, y \circ x=0) \Rightarrow x=y) \text {. }
\end{aligned}
$$

Definition $1.2([9])$. An algebra $A=(A, 0,0)$ of type $(2,0)$ is called a UP-algebra, where $A$ is a nonempty set, $\circ$ is a binary operation on $A$, and 0 is a fixed element of $A$ if it satisfies the following axioms: (1.1), (1.2), (1.3), and

$$
(\text { for all } x, y, z \in A)((y \circ z) \circ((x \circ y) \circ(x \circ z))=0) \text {. }
$$

From [9], we know that the concept of UP-algebras is a generalization of KU-algebras.

The binary relation $\leqslant$ on a UP-algebra $A=(A, 0,0)$ is defined as follows:

$$
(\text { for all } x, y \in A)(x \leqslant y \Leftrightarrow x \circ y=0)
$$

and the following assertions are valid (see $[9,10])$ :

$$
\begin{aligned}
& (\text { for all } x \in A)(x \circ x=0), \\
& (\text { for all } x, y, z \in A)((x \circ y=0, y \circ z=0) \Rightarrow x \circ z=0), \\
& (\text { for all } x, y, z \in A)(x \circ y=0 \Rightarrow(z \circ x) \circ(z \circ y)=0), \\
& (\text { for all } x, y, z \in A)(x \circ y=0 \Rightarrow(y \circ z) \circ(x \circ z)=0), \\
& (\text { for all } x, y, z \in A)(x \circ(y \circ x)=0, \text { in particular, }(y \circ z) \circ(x \circ(y \circ z))=0), \\
& (\text { for all } x, y \in A)((y \circ x) \circ x=0 \Leftrightarrow x=y \circ x), \\
& \text { (for all } x, y \in A)(x \circ(y \circ y)=0), \\
& (\text { for all } a, x, y, z \in A)((x \circ(y \circ z)) \circ(x \circ((a \circ y) \circ(a \circ z)))=0), \\
& (\text { for all } a, x, y, z \in A)((((a \circ x) \circ(a \circ y)) \circ z) \circ((x \circ y) \circ z)=0), \\
& (\text { for all } x, y, z \in A)(((x \circ y) \circ z) \circ(y \circ z)=0), \\
& \text { (for all } x, y, z \in A)(x \circ y=0 \Rightarrow x \circ(z \circ y)=0), \\
& \text { (for all } x, y, z \in A)(((x \circ y) \circ z) \circ(x \circ(y \circ z))=0), \text { and } \\
& \text { (for all } a, x, y, z \in A)(((x \circ y) \circ z) \circ(y \circ(a \circ z))=0) \text {. }
\end{aligned}
$$

Example 1.3 ([28]). Let $U$ be a nonempty set and let $X \in \mathcal{P}(U)$ where $\mathcal{P}(U)$ means the power set of $U$. Let $\mathcal{P}_{X}(\mathrm{U})=\{A \in \mathcal{P}(\mathrm{U}) \mid \mathrm{X} \subseteq A\}$. Define a binary operation $\triangle$ on $\mathcal{P}_{\mathbf{X}}(\mathrm{U})$ by putting $A \triangle B=B \cap\left(A^{C} \cup X\right)$ for all $A, B \in \mathcal{P}_{X}(U)$ where $A^{C}$ means the complement of a subset $A$. Then $\left(\mathcal{P}_{X}(U), \triangle, X\right)$ is a UP-algebra. Let $\mathcal{P}^{X}(U)=\{A \in \mathcal{P}(U) \mid A \subseteq X\}$. Define a binary operation $\Delta$ on $\mathcal{P}^{X}(U)$ by putting $A \Delta B=B \cup\left(A^{C} \cap X\right)$ for all $A, B \in \mathcal{P}^{X}(U)$. Then $\left(\mathcal{P}^{X}(U), \mathbf{\Lambda}, X\right)$ is a UP-algebra. 
Example 1.4 ([7]). Let $\mathbb{Z}^{*}$ be the set of all nonnegative integers. Define two binary operations $\circ$ and $\star$ on $\mathbb{Z}^{*}$ by:

$$
\left(\text { for all } m, n \in \mathbb{Z}^{*}\right)\left(m \circ n=\left\{\begin{array}{ll}
n, & \text { if } m<n, \\
0, & \text { otherwise, }
\end{array}\right)\right.
$$

and

$$
\text { (for all } \left.m, n \in \mathbb{Z}^{*}\right)\left(m \star n=\left\{\begin{array}{ll}
n, & \text { if } m>n \text { or } m=0, \\
0, & \text { otherwise. }
\end{array}\right)\right. \text {. }
$$

Then $\left(\mathbb{Z}^{*}, \circ, 0\right)$ and $\left(\mathbb{Z}^{*}, \star, 0\right)$ are UP-algebras.

For more examples and various studies of UP-algebras, see [1, 2, 10-12, 15, 27, 28, 30, 31, 33].

Definition $1.5([8])$. An algebra $A=(A, 0,0)$ of type $(2,0)$ is called a PU-algebra, where $A$ is a nonempty set, $O$ is a binary operation on $A$, and 0 is a fixed element of $A$ if it satisfies the axioms (1.1) and

$$
(\text { for all } x, y, z \in A)((x \circ z) \circ(y \circ z)=y \circ x) \text {. }
$$

From [8], we know that the concepts of KU-algebras and PU-algebras are different in general.

Definition 1.6. An algebra $A=(A, \circ, 0)$ of type $(2,0)$ is called an independent UP-algebra (or IUP-algebra for shot), where $A$ is a nonempty set, $\circ$ is a binary operation on $A$, and 0 is a fixed element of $A$ if it satisfies the axioms (1.1), (1.5), and

$$
(\text { for all } x, y, z \in A)((x \circ y) \circ(x \circ z)=y \circ z) .
$$

We define the binary relation $\leqslant$ on an IUP-algebra $A=(A, 0,0)$ as follows:

$$
\text { (for all } x, y \in A)(x \leqslant y \Leftrightarrow x \circ y=0) \text {. }
$$

Next, we'll give some interesting examples of IUP-algebras to support this new concept.

Example 1.7. Let $A=\{0,1,2,3,4,5\}$ be a set with a binary operation $\circ$ defined by the following Cayley table:

\begin{tabular}{l|llllll}
$\circ$ & 0 & 1 & 2 & 3 & 4 & 5 \\
\hline 0 & 0 & 1 & 2 & 3 & 4 & 5 \\
1 & 2 & 0 & 1 & 4 & 5 & 3 \\
2 & 1 & 2 & 0 & 5 & 3 & 4 \\
3 & 3 & 4 & 5 & 0 & 1 & 2 \\
4 & 4 & 5 & 3 & 2 & 0 & 1 \\
5 & 5 & 3 & 4 & 1 & 2 & 0
\end{tabular}

then $A=(A, \circ, 0)$ is an IUP-algebra. Since $(2 \circ 4) \circ(5 \circ 4)=3 \circ 2=5 \neq 4=5 \circ 2$, we have $A$ is not a PU-algebra.

Example 1.8. Let $(G, \circ, e)$ be a group such that all elements self-inverse. Then $(G, \circ, e)$ is an IUP-algebra but not a UP-algebra because it does not satisfy (1.2).

Example 1.9. Let $A$ be a set and $\mathcal{P}(A)$ means the power set of $A$. It follows from Example 1.8 that $(\mathcal{P}(A), \triangle, \emptyset)$ is an IUP-algebra where the binary operation $\triangle$ is defined as the symmetric difference of any two sets. 
Example 1.10. Let $\mathrm{G}$ be a group with the identity element $e$. Define a binary operation $\circ$ on $\mathrm{G}$ by:

$$
\text { (for all } x, y \in G)\left(x \circ y=y x^{-1}\right) \text {. }
$$

Then $(G, \circ, e)$ is an IUP-algebra but not a UP-algebra because it does not satisfy (1.2).

Example 1.11. Let $\mathbb{R}$ be the set of all real numbers. Define a binary operation $\circ$ on $\mathbb{R}$ by:

$$
\text { (for all } x, y \in \mathbb{R})(x \circ y=y-x) \text {. }
$$

It follows from Example 1.10 that $(\mathbb{R}, \circ, 0)$ is an IUP-algebra.

Example 1.12. Let $\mathbb{R}^{*}$ be the set of all nonzero real numbers. Define a binary operation $\circ$ on $\mathbb{R}^{*}$ by:

$$
\text { (for all } \left.x, y \in \mathbb{R}^{*}\right)\left(x \circ y=\frac{y}{x}\right) .
$$

It follows from Example 1.10 that $\left(\mathbb{R}^{*}, 0,1\right)$ is an IUP-algebra.

Example 1.13. Let $\mathbb{R}^{+}$be the set of all positive real numbers. Define a binary operation $\circ$ on $\mathbb{R}^{+}$by:

$$
\left(\text { for all } x, y \in \mathbb{R}^{+}\right)\left(x \circ y=\frac{y}{x}\right) .
$$

It follows from Example 1.10 that $\left(\mathbb{R}^{+}, \circ, 1\right)$ is an IUP-algebra.

Example 1.14. Let $A=\{0,1,2,3,4,5,6,7\}$ be a set with a binary operation $\circ$ defined by the following Cayley table:

\begin{tabular}{l|llllllll}
$\circ$ & 0 & 1 & 2 & 3 & 4 & 5 & 6 & 7 \\
\hline 0 & 0 & 1 & 2 & 3 & 4 & 5 & 6 & 7 \\
1 & 0 & 0 & 0 & 0 & 0 & 0 & 0 & 0 \\
2 & 0 & 1 & 0 & 0 & 0 & 0 & 0 & 0 \\
3 & 0 & 1 & 2 & 0 & 0 & 0 & 0 & 0 \\
4 & 0 & 1 & 2 & 3 & 0 & 0 & 0 & 0 \\
5 & 0 & 1 & 2 & 3 & 4 & 0 & 0 & 0 \\
6 & 0 & 1 & 2 & 3 & 4 & 5 & 0 & 0 \\
7 & 0 & 1 & 2 & 3 & 4 & 5 & 6 & 0
\end{tabular}

then $A=(A, \circ, 0)$ is a UP-algebra. Since $(2 \circ 7) \circ(2 \circ 6)=0 \circ 0=0 \neq 6=7 \circ 6$, we have $A$ is not an IUP-algebra.

In the previous examples, we know that the concepts of UP-algebras and IUP-algebras are different in general.

Proposition 1.15. In an IUP-algebra $A=(A, \circ, 0)$, the following assertions are valid:

(1) $($ for all $x, y \in A)((x \circ 0) \circ(x \circ y)=y)$;

(2) (for all $x \in A)((x \circ 0) \circ(x \circ 0)=0)$;

(3) $($ for all $x, y \in A)((x \circ y) \circ 0=y \circ x)$;

(4) (for all $x \in A)((x \circ 0) \circ 0=x)$;

(5) $($ for all $x, y \in A)(x \circ((x \circ 0) \circ y)=y)$;

(6) (for all $x, y \in A)(((x \circ 0) \circ y) \circ x=y \circ 0)$;

(7) (for all $x, y, z \in A)(x \circ y=x \circ z \Leftrightarrow y=z)$; 
(8) (for all $x, y \in A)(x \circ y=0 \Leftrightarrow x=y)$;

(9) (for all $x \in A)(x \circ 0=0 \Leftrightarrow x=0)$;

(10) (for all $x, y, z \in A)(y \circ x=z \circ x \Leftrightarrow y=z)$;

(11) (for all $x, y \in A)(x \circ y=y \Rightarrow x=0)$;

(12) $($ for all $x, y, z \in A)((x \circ y) \circ 0=(z \circ y) \circ(z \circ x))$;

(13) (for all $x, y, z \in A)(x \circ y=0 \Leftrightarrow(z \circ x) \circ(z \circ y)=0)$;

(14) (for all $x, y, z \in A)(x \circ y=0 \Leftrightarrow(x \circ z) \circ(y \circ z)=0)$; and

(15) the right and the left cancellation laws hold.

Proof.

(1). For all $x, y \in A$,

$$
\begin{aligned}
(x \circ 0) \circ(x \circ y) & =0 \circ y \\
& =y .
\end{aligned}
$$

(2). It follows from (1) by taking $y=0$.

(3). For all $x, y \in A$,

$$
\begin{aligned}
(x \circ y) \circ 0 & =(x \circ y) \circ(x \circ x) \\
& =y \circ x .
\end{aligned}
$$

(4). It follows from (3) and (1.1) by taking $y=0$.

(5). For all $x, y \in A$,

$$
\begin{aligned}
x \circ((x \circ 0) \circ y) & =((x \circ 0) \circ 0) \circ((x \circ 0) \circ y) \\
& =y .
\end{aligned}
$$

(6). For all $x, y \in A$,

$$
\begin{aligned}
((x \circ 0) \circ y) \circ x & =((x \circ 0) \circ y) \circ((x \circ 0) \circ 0) \\
& =y \circ 0 .
\end{aligned}
$$

(7). Assume that $x \circ y=x \circ z$. Then

$$
\begin{aligned}
y & =0 \circ y \\
& =(x \circ 0) \circ(x \circ y) \\
& =(x \circ 0) \circ(x \circ z) \\
& =0 \circ z \\
& =z .
\end{aligned}
$$

The converse is obvious.

(8). It follows from (7) and (1.5) by taking $z=x$.

The converse follows from (1.5).

(9). It follows from (7) and (1.5) by taking $y=0$ and $z=x$. 
(10). Assume that $y \circ x=z \circ x$. Then

$$
\begin{aligned}
x \circ y & =(y \circ x) \circ 0 \\
& =(z \circ x) \circ 0 \\
& =x \circ z .
\end{aligned}
$$

By (7), we have $y=z$.

The converse is obvious.

(11). It follows from (10) and (1.1) by taking $y=x, x=y$ and $z=0$.

(12). For all $x, y, z \in A$,

$$
\begin{aligned}
(x \circ y) \circ 0 & =y \circ x \\
& =(z \circ y) \circ(z \circ x) .
\end{aligned}
$$

(13). It follows from (1.7).

(14). For all $x, y, z \in A$,

$$
\begin{aligned}
x \circ y=0 & \Leftrightarrow x=y \\
& \Leftrightarrow x \circ z=y \circ z \\
& \Leftrightarrow(x \circ z) \circ(y \circ z)=0 .
\end{aligned}
$$

(15). It follows from (7) and (10).

Theorem 1.16. The binary relation $\leqslant$ on an IUP-algebra A defined by (1.8) is the diagonal relation.

Proof. It is straightforward by Proposition 1.15 (8).

\section{Special subsets of IUP-algebras}

In a PU-algebra $A=(A, \circ, 0)$, Elkabany et al. [8] defined the G-part and the P-radical of $A$, respectively, as follows:

$$
\begin{aligned}
& G(A)=\{x \in A \mid x \circ 0=x\}, \\
& B(A)=\{x \in A \mid x \circ 0=0\} .
\end{aligned}
$$

They proved that $G(A)$ and $B(X)$ are PU-subalgebras of $A$ (see Lemma 3.9 in [8]).

In a BCI-algebra $A=(A, 0,0)$, Borzooei and Zahiri [5] defined the P-semisimple part of $A$ as follows:

$$
P(A)=\{x \in A \mid(x \circ 0) \circ 0=x\} .
$$

The following example and theorems present the facts of the G-part, the P-radical, and the P-semisimple part in an IUP-algebra.

Example 2.1. From Example 1.7, we have $G(A)=\{0,3,4,5\}$. Since $4,5 \in G(A)$ but $4 \circ 5=1 \notin G(A)$, we have $G(A)$ is not an IUP-subalgebra of $A$.

Theorem 2.2. If $\mathrm{A}$ is an IUP-algebra, then $\mathrm{B}(\mathrm{A})=\{0\}$.

Proof. It is straightforward by Proposition 1.15 (9).

Theorem 2.3. If $\mathrm{A}$ is an IUP-algebra, then $\mathrm{P}(\mathrm{A})=\mathrm{A}$.

Proof. It is straightforward by Proposition 1.15 (4). 
For an IUP-algebra, we introduce new concepts of four special subsets: IUP-subalgebras, IUP-filters, IUP-ideals, strong IUP-ideals, as follows.

Definition 2.4. A nonempty subset $S$ of an IUP-algebra $A=(A, \circ, 0)$ is called

(1) an IUP-subalgebra of $A$ if it satisfies the following condition:

$$
\text { (for all } x, y \in S)(x \circ y \in S),
$$

(2) an IUP-filter (this concept uses the condition of UP-filters of UP-algebras) of A if it satisfies the following conditions:

$$
\begin{array}{r}
\text { the constant } 0 \text { of } A \text { is in } S \text {, } \\
\text { (for all } x, y \in A)(x \circ y \in S, x \in S \Rightarrow y \in S),
\end{array}
$$

(3) an IUP-ideal (this concept uses the condition of UP-ideals of UP-algebras) of $A$ if it satisfies the condition (2.2) and the following condition:

$$
\text { (for all } x, y, z \in A)(x \circ(y \circ z) \in S, y \in S \Rightarrow x \circ z \in S) \text {, }
$$

(4) a strong IUP-ideal (this concept uses the condition of near UP-filters of UP-algebras) of $A$ if it satisfies the following condition:

$$
(\text { for all } x, y \in A)(y \in S \Rightarrow x \circ y \in S) \text {. }
$$

We easily see that an IUP-algebra $A$ is an IUP-subalgebra, an IUP-filter, an IUP-ideal, and a strong IUP-ideal of $A$.

The following theorem is easy to verify.

Theorem 2.5. Let $\mathscr{U} \mathscr{S}$ be a nonempty family of IUP-subalgebras (resp., IUP-filters, IUP-ideals, strong IUP-ideals) of an IUP-algebra A. Then $\cap \mathscr{U} \mathscr{S}$ is an IUP-subalgebra (resp., IUP-filter, IUP-ideal, strong IUP-ideal) of A.

Theorem 2.6. $\{0\}$ is an IUP-ideal, an IUP-subalgebra, and an IUP-filter of an IUP-algebra A.

Proof. It is straightforward by (1.1), (1.5), and Proposition 1.15 (8).

Theorem 2.7. If $\mathrm{S}$ is an IUP-subalgebra of an IUP-algebra A, then it satisfies the condition (2.2).

Proof. It is straightforward by (1.5).

Theorem 2.8. A nonempty subset $S$ of an IUP-algebra $A=(A, 0,0)$ is a strong IUP-ideal of $A$ if and only if $S=A$.

Proof. Assume that $S$ is a strong IUP-ideal of $A$. Since $0 \in S$ and by Proposition 1.15 (4), we have $x=(x \circ 0) \circ 0 \in S$ for all $x \in A$. Hence, $S=A$.

The converse is obvious.

Theorem 2.9. Every IUP-ideal of an IUP-algebra $A=(A, 0,0)$ is an IUP-filter.

Proof. Assume that $S$ is an IUP-ideal of $A$. Then $S$ satisfies the condition (2.2). Next, let $x, y \in A$ be such that $x \circ y \in S$ and $x \in S$. By (1.1), we have $0 \circ(x \circ y)=x \circ y \in S$. By (2.4) and (1.1), we have $y=0 \circ y \in S$. Hence, $S$ is an IUP-filter of $A$.

Theorem 2.10. Every IUP-subalgebra of an IUP-algebra $A=(A, 0,0)$ is an IUP-filter.

Proof. Assume that $S$ is an IUP-subalgebra of $A$. By Theorem 2.7, we have $S$ satisfies the condition (2.2). Next, let $x, y \in A$ be such that $x \circ y \in S$ and $x \in S$. Since $0 \in S$ and by (2.1), we have $x \circ 0 \in S$. By (2.1), (1.7), and (1.1), we have $y=0 \circ y=(x \circ 0) \circ(x \circ y) \in S$. Hence, $S$ is an IUP-filter of $A$. 
Examples 2.11 and 2.12 show that the converse of Theorems 2.9 and 2.10 are not true in general, respectively.

Example 2.11. From Example 1.7, let $S=\{0,3\}$. Then $S$ is an IUP-subalgebra of $A$. By Theorem 2.10, we have $S$ is an IUP-filter of $A$. Since $3 \in S$ and $1 \circ(3 \circ 4)=1 \circ 1=0 \in S$ but $1 \circ 4=5 \notin S$, we have $S$ is not an IUP-ideal of $A$.

Example 2.12. From Example 1.12, let $S=\left\{x \in \mathbb{R}^{*} \mid x \geqslant 1\right\}$. Then $1 \in S$. Next, let $x, y, z \in \mathbb{R}^{*}$ be such that $x \circ(y \circ z) \geqslant 1$ and $y \geqslant 1$. Then $\frac{z}{y x} \geqslant 1$. Thus $x \circ z=\frac{z}{x}=\left(\frac{z}{y x}\right) y \geqslant 1$, that is, $x \circ z \in S$. Hence, $S$ is an IUP-ideal of $\mathbb{R}^{*}$. By Theorem 2.9, we have $S$ is an IUP-filter of $\mathbb{R}^{*}$. Since $1,2 \in S$ but $2 \circ 1=\frac{1}{2} \notin S$, we have $S$ is not an IUP-subalgebra of $\mathbb{R}^{*}$.

Theorem 2.13. Every strong IUP-ideal of an IUP-algebra A is an IUP-ideal and an IUP-subalgebra.

Proof. It is straightforward by Theorem 2.8 .

Examples 2.14 and 2.15 show that the converse of Theorem 2.13 is not true in general.

Example 2.14. From Example 1.7, let $S=\{0\}$. By Theorem 2.6, we have $S$ is an IUP-ideal of $A$. By Theorem 2.8 , we have $S$ is not a strong IUP-ideal of $A$.

Example 2.15. From Example 2.11, we have $S$ is an IUP-subalgebra of $A$. By Theorem 2.8, we have $S$ is not a strong IUP-ideal of $A$.

Examples 2.16 and 2.17 show that the concepts of IUP-ideals and IUP-subalgebras are not comparable in general.

Example 2.16. From Example 2.12, we have $S$ is an IUP-ideal of $\mathbb{R}^{*}$ but it is not an IUP-subalgebra of $\mathbb{R}^{*}$.

Example 2.17. From Example 2.11, we have $S$ is an IUP-subalgebra of $A$ but it is not an IUP-ideal of $S$.

By Theorems 2.9, 2.10, and 2.13 and Examples 2.11, 2.12, 2.14, and 2.15, we have that the concept of IUP-filters is a generalization of IUP-ideals and IUP-subalgebras, and IUP-ideals and IUP-subalgebras is a generalization of strong IUP-ideals. By Examples 2.16 and 2.17, we have that the concepts of IUPideals and IUP-subalgebras are not comparable in general. In an IUP-algebra $A$, we have that strong IUP-ideals can only be $A$ by Theorem 2.8. Then, we get the diagram of generalization of these concepts in UP-algebras as shown in Figure 1.

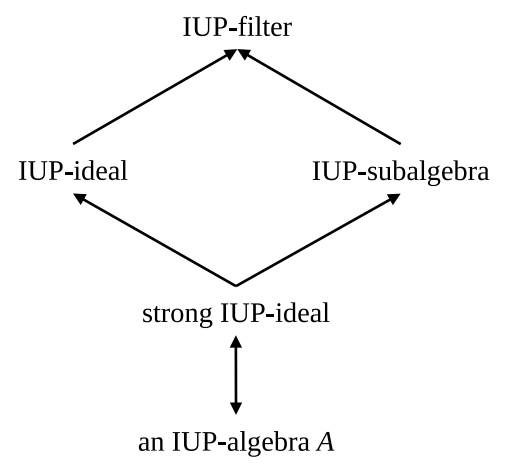

Figure 1: Four special subsets of IUP-algebras. 


\section{Homomorphisms of IUP-algebras}

In this section, we introduce the concept of homomorphisms between IUP-algebras as well as concepts of other algebra and study some characteristics of direct and inverse images of four special subsets.

Definition 3.1. Let $A=\left(A, 0,0_{A}\right)$ and $B=\left(B, *, 0_{B}\right)$ be IUP-algebras. A function $f: A \rightarrow B$ is called a homomorphism if it satisfies the following condition:

$$
(\text { for all } x, y \in A)(f(x \circ y)=f(x) * f(y)) \text {. }
$$

Example 3.2. Let $A=\left\{0_{A}, 1,2,3\right\}$ and $B=\left\{0_{B}, x, y, z\right\}$ be two IUP-algebras with fixed elements $0_{A}$ and $0_{B}$, and binary operations $\circ$ and $*$, respectively, defined by the following Cayley tables:

\begin{tabular}{|c|c|c|c|c|c|c|c|c|c|c|}
\hline 0 & $0_{A}$ & 1 & 2 & 3 & & $*$ & $0_{B}$ & $x$ & $y$ & $z$ \\
\hline $0_{\mathrm{A}}$ & $0_{A}$ & 1 & 2 & 3 & & $0_{\mathrm{B}}$ & $0_{B}$ & $x$ & $y$ & $z$ \\
\hline 1 & 1 & $0_{A}$ & 3 & 2 & and & $x$ & $y$ & $0_{B}$ & $z$ & $x$ \\
\hline 2 & 2 & 3 & $0_{\mathrm{A}}$ & 1 & & $y$ & $x$ & $z$ & $0_{B}$ & $y$ \\
\hline 3 & 3 & 2 & 1 & $0_{A}$ & & $z$ & $z$ & $y$ & $x$ & $0_{B}$ \\
\hline
\end{tabular}

We define a function $f: A \rightarrow B$ by:

$$
f=\left(\begin{array}{cccc}
0_{A} & 1 & 2 & 3 \\
0_{B} & z & z & 0_{B}
\end{array}\right)
$$

Then $f$ is a homomorphism.

Proposition 3.3. Let $\mathrm{A}=\left(\mathrm{A}, \mathrm{o}_{0} 0_{\mathrm{A}}\right)$ and $\mathrm{B}=\left(\mathrm{B}, *, 0_{\mathrm{B}}\right)$ be IUP-algebras, and $\mathrm{f}: \mathrm{A} \rightarrow \mathrm{B}$ be a homomorphism. Then the following assertions are valid:

(1) $f\left(0_{A}\right)=0_{B}$,

(2) (for all $x, y \in A)\left(f(x \circ y)=0_{B} \Leftrightarrow f(x)=f(y)\right)$;

(3) (for all $x \in A)\left(f(x)=0_{B} \Leftrightarrow f(x \circ 0)=0_{B}\right)$;

(4) (for all $x, y \in A)\left(x \circ y=0_{A} \Rightarrow f(x)=f(y)\right)$; and

(5) $\mathrm{f}^{-1}\left(\left\{0_{\mathrm{B}}\right\}\right)=\left\{0_{\mathrm{A}}\right\}$ if and only if $\mathrm{f}$ is injective.

Proof.

(1). By (1.5) and (3.1), we have $f\left(0_{A}\right)=f\left(0_{A} \circ 0_{A}\right)=f\left(0_{A}\right) * f\left(0_{A}\right)=0_{B}$.

(2). It is straightforward by (1) and Proposition 1.15 (8).

(3) and (4). It is straightforward by (1) and (2).

(5). It is straightforward by (2) and Proposition 1.15 (8).

Theorem 3.4. Let $\mathrm{A}=\left(\mathrm{A}, \mathrm{o}, 0_{\mathrm{A}}\right)$ and $\mathrm{B}=\left(\mathrm{B}, *, 0_{\mathrm{B}}\right)$ be IUP-algebras, and $\mathrm{f}: \mathrm{A} \rightarrow \mathrm{B}$ be a homomorphism. Then the following assertions are valid:

(1) if $\mathrm{S}$ is an IUP-subalgebra of $\mathrm{A}$, then the image $\mathrm{f}(\mathrm{S})$ is an IUP-subalgebra of $\mathrm{B}$, in particular, the image of $\mathrm{f}$ is an IUP-subalgebra of $\mathrm{B}$;

(2) if $\mathrm{S}$ is an IUP-subalgebra of $\mathrm{B}$, then the inverse image $\mathrm{f}^{-1}(\mathrm{~S})$ is an IUP-subalgebra of $\mathrm{A}$;

(3) if $S$ is a nonempty subset of $A$ such that $f^{-1}(f(S))$ is an IUP-filter of $A$, then the image $f(S)$ is an IUP-filter of $f(A)$;

(4) if $S$ is an IUP-filter of $A$ and $f$ is injective, then the image $f(S)$ is an IUP-filter of $f(A)$; 
(5) if $S$ is an IUP-filter of $B$, then the inverse image $f^{-1}(S)$ is an IUP-filter of $A$;

(6) if $S$ is a nonempty subset of $A$ such that $f^{-1}(f(S))$ is an IUP-ideal of $A$, then the image $f(S)$ is an IUP-ideal of $\mathrm{f}(\mathrm{A})$;

(7) if $\mathrm{S}$ is an IUP-ideal of $\mathrm{A}$ and $\mathrm{f}$ is injective, then the image $\mathrm{f}(\mathrm{S})$ is an IUP-ideal of $\mathrm{f}(\mathrm{A})$;

(8) if $\mathrm{S}$ is an IUP-ideal of $\mathrm{B}$, then the inverse image $\mathrm{f}^{-1}(\mathrm{~S})$ is an IUP-ideal of $\mathrm{A}$;

(9) if $\mathrm{S}$ is a strong IUP-ideal of $\mathrm{A}$ and $\mathrm{f}$ is surjective, then the image $\mathrm{f}(\mathrm{S})$ is a strong IUP-ideal of $\mathrm{B}$;

(10) if $\mathrm{S}$ is a strong IUP-ideal of $\mathrm{B}$, then the inverse image $\mathrm{f}^{-1}(\mathrm{~S})$ is a strong IUP-ideal of $\mathrm{A}$.

Proof.

(1). Assume that $S$ is an IUP-subalgebra of $A$. By Theorem 2.7 and by Proposition 3.3 (1), we have $0_{B}=f\left(0_{A}\right) \in f(S)$ is nonempty. Let $f(x), f(y) \in f(S)$ where $x, y \in S$. Then $x \circ y \in S$, so $f(x) * f(y)=$ $f(x \circ y) \in f(S)$. Hence, $f(S)$ is an IUP-subalgebra of $B$.

(2). Assume that $S$ is an IUP-subalgebra of B. By Theorem 2.7 and by Proposition 3.3 (1), we have $f\left(0_{A}\right)=0_{B} \in S$. Thus $0_{A} \in f^{-1}(S)$ is nonempty. Let $x, y \in f^{-1}(S)$. Then $f(x), f(y) \in S$. By (2.1), we have $f(x \circ y)=f(x) * f(y) \in S$. Thus $x \circ y \in f^{-1}(S)$. Hence, $f^{-1}(S)$ is an IUP-subalgebra of $A$.

(3). Assume that $S$ is a nonempty subset of $A$ such that $f^{-1}(f(S))$ is an IUP-filter of $A$. By (2.2) and Proposition $3.3(1)$, we have $0_{B}=f\left(0_{A}\right) \in f(S)$. Let $f(x), f(y) \in f(A)$ be such that $f(x) * f(y) \in f(S)$ and $f(x) \in f(S)$. Then $f(x \circ y) \in f(S)$. Thus $x \circ y \in f^{-1}(f(S))$ and $x \in f^{-1}(f(S))$. By $(2.3)$, we have $y \in f^{-1}(f(S))$. Thus $f(y) \in f(S)$. Hence, $f(S)$ is an IUP-filter of $f(A)$.

(4). Assume that $S$ is an IUP-filter of $A$. Since $f$ is injective, we have $f^{-1}(f(S))=S$ is an IUP-filter of $A$.

(5). Assume that $S$ is an IUP-filter of B. By (2.2) and Proposition $3.3(1)$, we have $f\left(0_{A}\right)=0_{B} \in S$. Thus $0_{A} \in f^{-1}(S)$. Let $x, y \in A$ be such that $x \circ y \in f^{-1}(S)$ and $x \in f^{-1}(S)$. Then $f(x) * f(y)=f(x \circ y) \in S$ and $f(x) \in S$. By (2.3), we have $f(y) \in S$. Thus $y \in f^{-1}(S)$. Hence, $f^{-1}(S)$ is an IUP-filter of $A$.

(6). Assume that $S$ is a nonempty subset of $A$ such that $f^{-1}(f(S))$ is an IUP-ideal of $A$. By (2.2) and Proposition $3.3(1)$, we have $0_{B}=f\left(0_{A}\right) \in f(S)$. Let $f(x), f(y), f(z) \in f(A)$ be such that $f(x) *(f(y) * f(z)) \in$ $f(S)$ and $f(y) \in f(S)$. Then $f(x \circ(y \circ z)) \in f(S)$. Thus $x \circ(y \circ z) \in f^{-1}(f(S))$ and $y \in f^{-1}(f(S))$. By (2.4), we have $x \circ z \in f^{-1}(f(S))$. Thus $f(x) * f(z)=f(x \circ z) \in f(S)$. Hence, $f(S)$ is an IUP-ideal of $f(A)$.

(7). Assume that $S$ is an IUP-ideal of $A$. Since $f$ is injective, we have $f^{-1}(f(S))=S$ is an IUP-ideal of $A$.

(8). Assume that $S$ is an IUP-ideal of B. By (2.2) and Proposition $3.3(1)$, we have $f\left(0_{A}\right)=0_{B} \in S$. Thus $0_{A} \in f^{-1}(S)$. Let $x, y, z \in A$ be such that $x \circ(y \circ z) \in f^{-1}(S)$ and $y \in f^{-1}(S)$. Then $f(x) *(f(y) * f(z))=$ $f(x \circ(y \circ z)) \in S$ and $f(y) \in S$. By (2.4), we have $f(x \circ z)=f(x) * f(z) \in S$. Thus $x \circ z \in f^{-1}(S)$. Hence, $f^{-1}(S)$ is an IUP-ideal of $A$.

(9) and (10). It is straightforward by Theorem 2.8.

\section{Open problem}

Find an example that shows that Theorems $3.4(4)$ and (7) are not true if a homomorphism $f$ is not injective.

\section{Conclusions and future works}

We introduced the concept of a new algebraic structure: IUP-algebras; the study showed, this new algebraic structure is independent of UP-algebras. We have also introduced the concept of four special subsets, namely IUP-subalgebras, IUP-filters, IUP-ideals, and strong IUP-ideals of IUP-algebras, wherethe 
relationship of these four subsets is shown in Figure 1. Finally, we have presented and studied some characteristics of homomorphisms between IUP-algebras and obtained the results of the direct and inverse images of four special subsets, as in Theorem 3.4.

The research topics of interest by our research team being studied in IUP-algebras are as follows:

(1) to study rough set theory based on the concept of Klinseesook et al. [17];

(2) to study fuzzy set theory (with respect to a triangular norm) based on the concept of Somjanta et al. [32] and Thongarsa et al. [6,33];

(3) to study bipolar fuzzy set theory based on the concept of Muhiuddin [19];

(4) to study interval-valued fuzzy set theory based on the concept of Muhiuddin et al. [20];

(5) to study Pythagorean fuzzy set theory based on the concept of Satirad et al. [25];

(6) to study picture fuzzy set theory based on the concept of Yuphaphin et al. [35]; and

(7) to study soft set theory of fuzzy sets based on the concept of Satirad and Iampan [26].

\section{Acknowledgment}

This work was supported by the revenue budget in 2021, School of Science, University of Phayao.

\section{References}

[1] M. A. Ansari, A. Haidar, A. N. A. Koam, On a graph associated to UP-algebras, Math. Comput. Appl., 23 (2018), 12 pages. 1

[2] M. A. Ansari, A. N. A. Koam, A. Haider, Rough set theory applied to UP-algebras, Ital. J. Pure Appl. Math., 42 (2019), 388-402. 1

[3] R. K. Bandaru, N. Rafi, A. Rezaei, On eGE-algebras, Discuss. Math. Gen. Algebra Appl., 41 (2021), 395-409. 1

[4] R. A. Borzooei, A. Borumand Saeid, A. Rezaei, A. Radfar, R. Ameri, On pseudo-BE-algebras, Discuss. Math., Gen. Algebra Appl., 33 (2013), 95-108. 1

[5] R. A. Borzooei, O. Zahiri, Prime ideals in BCI and BCK-algebras, Ann. Univ. Craiova Math. Comput. Sci. Ser., 39 (2012), 266-276. 2

[6] P. Burandate, S. Thongarsa, A. Iampan, Fuzzy sets in UP-algebras with respect to a triangular norm, Konuralp J. Math., 7 (2019), 410-432. (2)

[7] N. Dokkhamdang, A. Kesorn, A. Iampan, Generalized fuzzy sets in UP-algebras, Ann. Fuzzy Math. Inform., 16 (2018), 171-190. 1.4

[8] A. E. I. Elkabany, M. A. Abdel Naby, S. M. Mostafa, New view of ideals on PU-algebra, Int. J. Comput. Appl., 111 (2015), 9-15. 1.5, 1, 2

[9] A. Iampan, A new branch of the logical algebra: UP-algebras, J. Algebra Relat. Topics, 5 (2017), 35-54. 1, 1.2, 1, 1

[10] A. Iampan, Introducing fully UP-semigroups, Discuss. Math. Gen. Algebra Appl., 38 (2018), 297-306. 1, 1

[11] A. Iampan, Multipliers and near UP-filters of UP-algebras, J. Discrete Math. Sci. Cryptogr., 24 (2021), 667-680.

[12] A. Iampan, M. Songsaeng, G. Muhiuddin, Fuzzy duplex UP-algebras, Eur. J. Pure Appl. Math., 13 (2020), $459-471$. 1

[13] Y. Imai, K. Iséki, On axiom systems of propositional calculi. XIV, Proc. Japan Acad., 42 (1966), 19-22. 1

[14] K. Iséki, An algebra related with a propositional calculus, Proc. Japan Acad., 42 (1966), 26-29. 1

[15] Y. B. Jun, G. Muhiuddin, D. A. Romano, On filters in UP-algebras, a review and some new reflections, J. Int. Math. Virtual Inst., 11 (2021), 35-52. 1

[16] H. S. Kim, Y. H. Kim, On BE-algebras, Sci. Math. Japon., 66 (2007), 113-116. 1

[17] T. Klinseesook, S. Bukok, A. Iampan, Rough set theory applied to UP-algebras, J. Inform. Optim. Sci., 41 (2020), 705-722. (1)

[18] P. Mosrijai, A. Iampan, A new branch of bialgebraic structures: UP-bialgebras, J. Taibah Univ. Sci., 13 (2019), 450-459. 1

[19] G. Muhiuddin, Bipolar fuzzy KU-subalgebras/ideals of KU-algebras, Ann. Fuzzy Math. Inform., 8 (2014), 409-418. (3)

[20] G. Muhiuddin, D. Al-Kadi, A. Mahboob, More general form of interval-valued fuzzy ideals of BCK/BCI-algebras, Secur. Commun. Netw., 2021 (2021), 10 pages. (4)

[21] C. Prabpayak, U. Leerawat, On ideals and congruences in KU-algebras, Sci. Magna, 5 (2009), 54-57. 1, 1.1 
[22] A. Rezaei, A. B. Saeid, A. Radfar, On eBE-algebras, TWMS J. Pure Appl. Math., 7 (2016), 200-210. 1

[23] A. Rezaei, A. B. Saeid, K. Y. S. Saber, On pseudo-CI-algebras, Soft Comput., 23 (2019), 4643-4654. 1

[24] A. Satirad, R. Chinram, A. Iampan, Four new concepts of extensions of KU/UP-algebras, Missouri J. Math. Sci., 32 (2020), 138-157. 1

[25] A. Satirad, R. Chinram, A. Iampan, Pythagorean fuzzy sets in UP-algebras and approximations, AIMS Math., 6 (2021), 6002-6032. (5)

[26] A. Satirad, A. Iampan, Fuzzy soft sets over fully UP-semigroups, Eur. J. Pure Appl. Math., 12 (2019), 294-331. (7)

[27] A. Satirad, P. Mosrijai, A. Iampan, Formulas for finding UP-algebras, Int. J. Math. Comput. Sci., 14 (2019), $403-409$. 1

[28] A. Satirad, P. Mosrijai, A. Iampan, Generalized power UP-algebras, Int. J. Math. Comput. Sci., 14 (2019), 17-25. 1.3, 1

[29] S. Sayyad, H. Babaei, A. Rezaei, On pseudo-eBE-algebras, Soft Comput., 24 (2020), 7005-7020. 1

[30] T. Senapati, Y. B. Jun, K. P. Shum, Cubic set structure applied in UP-algebras, Discrete Math. Algorithms Appl., 10 (2018), 23 pages. 1

[31] T. Senapati, G. Muhiuddin, K. P. Shum, Representation of UP-algebras in interval-valued intuitionistic fuzzy environment, Ital. J. Pure Appl. Math., 38 (2017), 497-517. 1

[32] J. Somjanta, N. Thuekaew, P. Kumpeangkeaw, A. Iampan, Fuzzy sets in UP-algebras, Ann. Fuzzy Math. Inform., 12 (2016), 739-756. (2)

[33] S. Thongarsa, P. Burandate, A. Iampan, Some operations of fuzzy sets in UP-algebras with respect to a triangular norm, Ann. Commun. Math., 2 (2019), 1-10. 1, (2)

[34] P. Yiarayong, P. Wachirawongsakorn, A new generalization of BE-algebras, Heliyon, 4 (2018), 11 pages. 1

[35] S. Yuphaphin, P. Kankaew, N. Lapo, R. Chinram, A. Iampan, Picture fuzzy sets in UP-algebras by means of a special type, J. Math. Comput. Sci., 25 (2022), 37-72. (6) 